\title{
La expansión de la academia. Perspectivas, problemas y prácticas en la investigación artística local.
}

\author{
Francisca García Barriga \\ Universidad Metropolitana de Ciencias de la Educación \\ mfranciscagarcia@gmail.com
}

\author{
Ignacio Nieto Larraín \\ Universidad Finis Terrae \\ ignacio.nieto@ug.uchile.cl \\ Artículo bajo licencia Creative Commons \\ Atribución 4.0 Internacional (CC BY 4.0) \\ ENVIADO: 20-09-29 \\ ACEPTADO: $20-12-22$
}

\section{RESUMEN}

En el presente artículo proponemos un estado del arte respecto del aparato conceptual internacional asociado a la "investigación artística”, en diálogo con aproximaciones locales de investigadores chilenos. Algunas de las cuestiones que abordamos refieren a la multiplicidad de formas de investigación artística en la producción de conocimiento, a su dimensión epistemológica e interdisciplinar. Junto a ello, revisamos las condiciones materiales para su desarrollo en Chile y los programas de postgrado emergentes en las universidades chilenas que orientan la práctica a este tipo de investigación. El trabajo incluye tres “insertos artísticos” que permitirán al lector acercarse a diferentes metodologías de trabajo desde una lógica teórico-práctica. Finalmente se dan ciertas recomendaciones según lo presentado y de acuerdo a la experiencia obtenida a partir de seminarios impartidos por los autores.

Palabras clave

Práctica artística como investigación; Academia; Interdisciplina; Artes Visuales; Afectos.

\section{RESUMO}

Neste artigo propomos um estado da arte sobre o aparato conceitual internacional associado à "investigação artística", em diálogo com as abordagens dos pesquisadores chilenos. Algumas das questões que respondemos referem-se à investigação artística na produção do conhecimento, a sua dimensão epistemológica e interdisciplinar. Junto com isso, fazemos uma revisão da matriz legislativa e dos programas de pós-graduação emergentes em universidades chilenas para o desenvolvimento desse tipo de pesquisa. Ao mesmo tempo, a obra inclui três "inserções artísticas" que permitirão ao leitor abordar diferentes metodologias de trabalho a partir de uma lógica teórico-prática. Por fim, algumas recomendações são apresentadas de acordo com a experiência obtida nos seminários ministrados orientados para esta linha de investigação.

PALAVRAS-CHAVE

Investigação artística; Academia; Interdisciplina; Artes visuais; Afetos.

\section{ABSTRACT}

The article provides an international state of the art for the "artistic research", including local approaches by Chilean researchers. Some of the questions that we answer refer to artistic research in the context of production of knowledge and its epistemological and interdisciplinary dimension. Then we review the material conditions for its development in Chile and the emerging postgraduate programs in Chilean universities that guide the practice of this kind of research. The paper includes three "artistic inserts" that will allow the reader to approach different work methodologies from a theoretical-practical point of view. Finally, certain recommendations are given by the authors in accordance with the experience obtained from teaching and given seminars.

KEYWORDS

Arts Based Research; Academy; Interdiscipline; Visual Arts; Affects. 


\section{DisCUSIÓN CONCEPTUAL}

Lo que se llama "percepción» ya no es un estado de cosas, sino un estado del cuerpo en tanto que inducido por otro cuerpo; $y$ «afección» es el paso de este estado a otro en tanto que aumento o disminución del exponente-potencia, bajo la acción de otros cuerpos ninguno es pasivo, sino que todo es interacción, incluso la gravedad.

Deleuze \& Guattari, Qu'est-ce que la philosophie? (1991).

El notorio anarquismo se concibe sobre todo como una terapia contra las consecuencias antidemocráticas y opresivas del monopolio de la autoridad epistémica que mantiene la ciencia.

Feyerabend, Against Method (1975).

La crisis del conocimiento disciplinar en la actualidad atraviesa el sistema académico en una dimensión global y en todas las facultades universitarias. Funcionarios, investigadores, académicos y estudiantes estamos desafiados a pensar de forma cooperativa y más allá de las fronteras del conocimiento adquirido en las fases de la educación formal. Este desafío, que es teórico y práctico a la vez, se relaciona estrechamente con algunas discusiones vigentes hoy en torno a la práctica artística en el espacio académico, respecto de su rol y naturaleza, su legitimación en la producción del conocimiento, su financiamiento y proyección futura en diálogo con la educación. A modo preliminar es posible establecer que la práctica artística busca hoy multiplicar los espacios para el trabajo intelectual crítico, cuestionando los sistemas dominantes y con una actitud interdisciplinaria (Wilson y Van Ruiten, 2013: 9).

Se sabe que la "investigación artística" no es algo nuevo, en la medida que en todas las épocas los artistas han reflexionado e investigado bajo diversas metodologías a partir de problemas, materiales, técnicas y referentes. Sin embargo, la legitimación del conocimiento artístico en el corazón mismo del sistema científico-tecnológico -que es el que rige el desarrollo de conocimiento e investigación en la academia- supone hoy la novedad y el desafío. En este marco, la batalla de la investigación artística hace frente también a la fetichización de la "obra", la interpretación semiológica del objeto y su encasillamiento como objeto cultural, que han sido las perspectivas más tradicionales con que la ciencia y el logos han impulsado sus diálogos hacia el campo artístico. De este modo las investigaciones basadas en la práctica subrayan que el arte es una forma de epistemología en sí, que trasciende la experiencia puramente estética y de carácter interpretativo (Eisner, 2006: 10).

Las debilidades que hoy exhibe la lógica disciplinar y separatista del conocimiento contrastarían con la lógica relacional e interactiva que impulsa la epistemología artística. Numerosa bibliografía ha comprobado que la práctica artística efectivamente produce conocimiento (Sullivan, 2010; Barone y Elliot, 2012; Savin-Baden y Wimpenny, 2014). Uno de los antecedentes que abre la discusión es el libro de 1934 de John Dewey, Art as an Experience. La teoría filosófica expuesta por Dewey es un intento de cambiar la comprensión de lo que es esencial y característico del proceso del arte, desde sus manifestaciones físicas en el objeto expresivo hasta el proceso en su totalidad, un proceso cuyo elemento fundamental ya no es la obra de arte material sino el desarrollo de una experiencia, experiencia mediada por una perspectiva crítica de la percepción (Dewey citado por Leddy 2020).

Considerando este antecedente, las prácticas artísticas no son autónomas, sino situadas e incrustadas. El significado del arte se genera en las interacciones de entornos experimentales que son un medio para influir en los pensamientos y actos del practicante. Este tipo de aproximación contrasta con la investigación científica ya que provoca una distinción que, según Cazeaux (2018), proviene del razonamiento emanado de la experiencia humana. El autor argumenta que la investigación artística o la práctica del artista es definida por la experiencia: "yo tengo la experiencia del mundo como una secuencia organizada de fenómenos, que reco- 
nozco, a partir de un entendimiento adquirido, interactúo con él. A partir de esto yo soy consciente de mí mismo, soy un ser que existe continuamente" (2018: 119).

Esa experiencia consciente no sólo está centrada en el objeto de estudio sino que en otros elementos subjetivos. Knowles y Cole (2008) identifican que la práctica artística está muchas veces cargada de elementos emocionales y se relacionan con experiencias afectivas, donde la imaginación y el intelecto dan formas de conocer y responder al mundo. Desde esa perspectiva, Lincoln y Denzin comentan que la práctica investigativa "implica procesos de descubrimiento y la invención de carácter crítico ya que son los momentos de epifanía en la cultura suspendidos en el tiempo. Estas abren las instituciones, para la inspección y evaluación de sus prácticas" (2003: 377). Siguiendo esta línea argumentativa, el investigador de la Universidad de Valparaíso Gustavo Celedón señala que el conocimiento del arte más bien "hace" y no "dice", y de ese "hacer" derivan las transferencias, los desvíos y los movimientos que genera toda experiencia. El mismo autor da cuenta que al abrir un ejercicio de pensamiento, hemos abierto antes incluso un despliegue de sentires. La investigación artística parece tener esta doble impronta, del sentir y el pensar (Celedón, 2020: 34).

En respuesta a la supremacía del logos moderno, la investigación artística también se ha pensado en relación al sistema científico dominante. Desde esa perspectiva es posible agenciar lo artístico como un activo en la producción de conocimiento, en la renovación de métodos de investigación, en la formación integral de personas y la generación de espacios de saber abiertos y más inclusivos. La práctica artística se pone en relación con el conocimiento científico, ya que ambas requieren lo que Rheinberger denomina como "sistema experimental" (1998). Esta noción remite a la actividad experiencial que combina aspectos locales, técnicos, instrumentales, institucionales, sociales y epistémicos. Estos aspectos no son atribuidos a la concepción moderna del arte, la cual identifica a la "obra" como un objeto autónomo, autocontenido a partir de una experiencia sensorial (Cazeaux, 2018).

La actriz, performer y académica de la Pontificia Universidad Católica de Chile María José Contreras ha llamado la atención sobre la necesidad de incorporar la conjunción de investigación y práctica artística en el marco de la academia chilena. Se refiere en particular a la metodología emergente que suele denominarse "arts based research" o "practice-based research" desde la tradición anglosajona y de países nórdicos, que ella propone llamar en el contexto latinoamericano "práctica como investigación". Este giro epistémico, señala Contreras, "ha conllevado una validación del cuerpo como agente activo del conocimiento" (2013: 73) y "las preguntas o motivaciones iniciales solo puedan ser contestadas mediante la práctica. Esto implica que si bien los aspectos conceptuales y la reflexión crítica están presentes, lo crucial sigue siendo la práctica" (2013: 76). Contreras realza además las transformaciones que ha experimentado la educación en Artes Visuales en Chile desde los años 90. Hasta ese momento la escritura y la investigación se entendían como una tarea secundaria exclusivamente vinculada a la "búsqueda de referentes" y a la redacción de "memorias de obra" supeditadas a la actividad de taller (Contreras, 2018: s/p). La autora señala que esta subordinación comienza a diluirse con la aparición de proyectos que, en el siglo XXI, asumen con mucho riesgo la integración de la "teoría" y la "praxis", entendidas ambas como fuerzas recíprocas y complementarias en la producción del conocimiento artístico.

Respecto de su potencial interdisciplinario, Carter ha señalado que el conocimiento adquirido en la experiencia práctica se articula integrando elementos ajenos a su norma disciplinar, más allá del virtuosismo en el manejo de las herramientas técnicas específicas (2011: 16). Esta premisa activa en la actualidad una pugna no solo en la definición de "obra" sino, sobre todo, en la definición misma de conocimiento, propiedad adjudicada tradicionalmente de forma exclusiva al saber científico (Nieto y Velasco, 2016; Sormani y Garbone, Proska, 2019). Este giro epistemológico concibe a las prácticas artísticas como investigación y considera sus metodologías poniéndolas en relación con otras disciplinas que se fundamentan con enfoques científicos, como podrían ser, por ejemplo, el arteterapia, la antropología, la etnografía, la educación artística, entre otros (Knowels y Cole, 2007; Schwab, 2013; Savin-Baden y Wimpenny, 2014). Se podría decir entonces que esta perspectiva validaría la teoría del "pluralismo científico" desarrollada por la denominada Escuela de Stanford, donde John Dupré, Ian Hacking, Peter Galison, Patrick Suppes y Nancy Cartwright consideran que un fenómeno estudiado por la ciencia puede ser explicado por más de una disciplina, por lo que existiría una polifonía de interpretaciones (Kellert, et al 2006) y por ende, existiría una crítica a la noción reduccionista del quehacer científico.

Existe una variedad de interpretaciones de la práctica artística como investigación. Carter (2004) apela a la interdisciplinariedad pues considera que uniendo diversas habilidades, experiencias e intereses, y conectando cosas dispares (lo cognitivo, lo fenomenológico y lo metafísico), se materializa el discurso en sí mismo. Hannula, Suoranta y Vadén (2005), por otro lado, defienden la investigación artística enfatizando que esta es una afirmación recurrente a un círculo hermenéutico, círculo que es un marco de referencias al que continuamente accedemos. Knowels y Cole (2007) se identifican con una aproximación antropológica, limitándose a técnicas cualitativas en combinación con formas de representaciones de diferente índole que van desde el teatro y la escritura creativa hasta los medios digitales. Schwab (2013) se enfoca en los vínculos entre los experimentos y la práctica artística, comparando el laboratorio con el estudio, centrándose en la práctica material, describiendo los sistemas de creación o destacando las dimensiones temporales o experienciales de la producción. Nieto y Velasco (2016) se enfocan por el cruce interdisciplinar y transdisciplinar, analizando metodologías y resultados obtenidos, realizando un contrapunto con diferentes formas de conocer definidas anteriormente por algunos filósofos como podrían ser Foucault o Goodman. Jae Emerling (2019) cuestiona la historicidad del arte, en cuanto esta no recurre a la experimentación sino que es una instancia donde se reconocen interpretaciones que son negativas a la experimentación -genealógicas diríamos-, de lo que deriva que la práctica debería escapar de su condición histórica. De acuerdo a esto, Borgdorff argumenta: "el contexto en el que tiene lugar la investigación artística está formado tanto por el mundo del arte como por el discurso académico; la relevancia de los temas y la validez de los resultados se so- 
pesan en el acontecimiento y a la luz de ambos contextos" (2011: 47).

Finalmente, un contrapunto a toda esta discusión y abanico de interpretaciones en torno a investigación basada en práctica, será la realizada por Doherty (2020). Desde una perspectiva "culturalista", el autor cuestiona el conocimiento dado por los sistemas de reproducción en entornos académicos ya que estos serían eminentemente colonialistas. Por ello considera integrar otro tipos de saberes -como por ejemplo, el vudú-, levantando la pregunta, “¿en el marco institucional de la universidad, deben las artes creativas, producir conocimientos de igual manera como lo hacen las ciencias o incluso las ciencias sociales?" (Doherty, 2020: IV; Adjei, 2020). Doherty abre con ello el campo de acción hacia otras formas de conocer mediante la práctica artística y los saberes que los rodean, constatando que las epistemologías y los lenguajes son también culturales y, por lo tanto, múltiples y simultáneos

\section{INSERTO INVESTIGACIÓN ARTÍSTICA \# 1}

Primates Cinema. Apes as Family (2011)

Dirección: Rachel Mayeri' ${ }^{1}$.

País: Reino Unido/ España-Islas Baleares. Productores: Steve Pallrand y Gillean Dickie.

Cámara: Ian Dodds, Rachel Mayeri y Steve Pallrand.

Editor: Rachel Mayeri.

Link:

https://www.artscatalyst.org/primate-cinema-apes-family

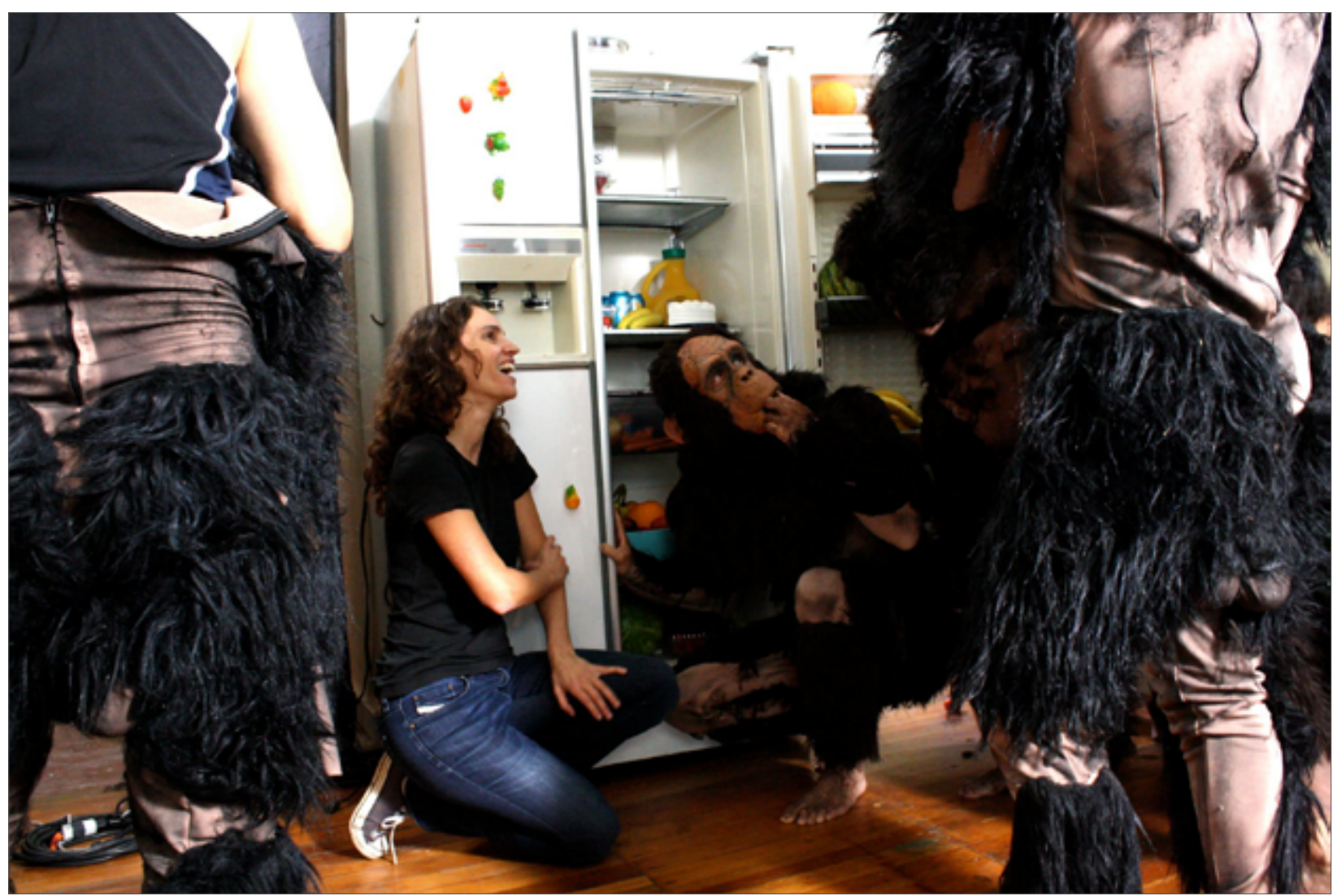

Imagen 1. Cortesía de Rachel Mayerì.

Making Off de Primates Cinema. Apes as Family, 2011, film de Rachel Mayeri. 
Película de ficción expuesta en la fosa de chimpancés del Zoológico de Edimburgo y posteriormente exhibida en festivales de arte y de cine, en varios países de Europa y América. Cortesía de Rachel Mayeri.

"Han pasado muchos años desde que realicé Apes as Family, y sigue siendo la pieza más ambiciosa, improbable y más colaborativa en la que he participado. El proyecto en su forma más simple era hacer una película expresamente para un público de chimpancés - y grabar a los chimpancés viendo la película. A los chimpancés se les muestra en cautiverio en video como una forma de enriquecimiento, pero ningún artista había hecho una película para ellos. La idea, tan emocionante al principio, era considerar otras mentes y tal vez reflejar esas mentes de una manera comprensible para los primates a través de la realización de la película.

En el proceso hubieron muchas discusiones sobre la investigación. Apes as Family surgió con el aumento al apoyo para la realización de proyectos que se relacionan arte-ciencia y de muchos años de compromiso como artista con los primates y los primatólogos. Mi primer trabajo en torno a esta investigación fue Baboons as Friends, una película como de cine negro, donde trabajé con una primatóloga que se convirtió en una colaboradora de larga data, Deborah Forster. Realizamos un taller sobre cómo actuar como un animal, con actores y no actores aprendiendo sobre la sociabilidad y el movimiento de los primates. Asistí a muchas conferencias de primatología y participé en muchas exposiciones. Después de eso, trabajé con babuinos, monos, ardillas, con cuidadores y científicos en un centro de cría en Francia. En ese momento, pude proponer el proyecto de hacer películas para chimpancés a Arts Catalyst por medio de The Wellcome Trust2. Mi interés en los primates había evolucionado desde las representaciones mediáticas a las comparaciones entre especies y las mentes de animales no humanos.

Esta idea se inspiró quizás en un artículo -más tarde en un libro- escrito por Charles Siebert "Planet of the Retired Chimps" (2005). Siebert conoció a un chimpancé en una residencia de ancianos, cuyo programa de televisión favorito era "Emergency Room" (Sala de Urgencias). En ese tiempo, me preguntaba si las camillas o las batas de laboratorio eran atractivas, ya que muchos de los chimpancés, que conocí habían sido criados en laboratorios. Mi primer pensamiento fue, entonces, hacer un drama de hospital para chimpancés. Pero el psicólogo con el que trabajaba en el zoológico de Edimburgo, sabiamente, me disuadió de tocar cualquier material morboso: los zoológicos, particularmente en el Reino Unido, son muy sensibles a los medios negativos. Más allá de esa primera idea, quería aprender lo que a los chimpancés les gustaba ver. De ahíque le pedí a muchos guardias de zoológico que me contaran sus historias. Investigué todos los artículos sobre el enriquecimiento de los chimpancés. Me acerqué al problema de cómo trabajar con chimpancés desde la perspectiva del investigador de mercado. De hecho lla-

Arts Catalyst (Reino Unido) se encarga del arte y la investigación transdisciplinaria para activar nuevas ideas, conversaciones y experiencias transformadoras a través de la ciencia y la cultura. Apoya proyectos que son financiados por el Art Council inglés. mé a uno para preguntarle si el proceso aplicaba: la respuesta fue no, porque no puedes entrevistarlos.

Estuve una semana grabando en vídeo a los chimpancés. Después trabajé con actores en mi taller sobre cómo actuar como chimpancés, e hicimos parodias de todos los dramas de la vida de los chimpancés: demostraciones de dominio, sexo, alimentación, uso de máscaras, visitas al médico. Hice un video de los chimpancés en el zoológico de Los Ángeles. Recolecté material: programas de cocina, películas de vida silvestre, caricaturas, tambores y anuncios con un chimpancé animatrónico. Montamos todo ese material en dos monitores en la cápsula de investigación en el zoológico de Edimburgo, con la idea de que pudiéramos determinar la preferencia por el monitor con el que los chimpancés pasaban más tiempo. Los chimpancés estaban muy emocionados al principio de que un nuevo grupo de investigadores estaba allí. Cuando estábamos observando su comportamiento, nos preguntábamos qué tipo de estímulos necesitarían los chimpancés: ¿aros para saltar y pasar a través de ellos? ¿comida?. Después del ritual de iniciación, se mostraron y me escupieron, y luego se acomodaron en unos pocos videos, realmente expresivos sobre su interés, pegando su cabeza muy cerca de la pantalla saltando de arriba hacia abajo con el pelo erecto, gritando.

El guión y la producción fueron estresantes. Necesitaba crear una historia con un elenco de chimpancés y por supuesto, eso sería imposible. Emplear chimpancés reales como actores no es ético. La animación $3 D$ es prohibitivamente cara. Pudimos un día con un actor utilizar un traje de chimpancé animado electro mecánicamente, cuyas expresiones faciales eran controladas en parte por dos operadores. Un chimpancé por sí mismo, no podría abordar ninguna situación social, por lo tanto, decidí que mi elenco de actores, hubiera un chimpancé "real" y varios chimpancés "falsos". Me refiero al chimpancé "real" criado en un hogar humano y luego abandonado; entonces conoce a un grupo de chimpancés falsos que sutilmente lo convencen de regresar a la naturaleza. La historia se inspiró en la lectura de "Next of Kin" de Roger Fouts, sobre el experimento de comunicación con lenguaje de señas con la chimpancé Washoez, quien como otros chimpancés enculturados, quedó entre el humano y el chimpancé en cuanto a comportamiento e identidad. Sentí que eso podría resonar con los chimpancés en cautiverio. La película consistiría en momentos emocionantes tanto para los chimpancés como para los humanos. El proceso continuó con la presentación de la película terminada de 22 minutos para los chimpancés y la grabación de su reacción. El proyecto se presentó como una instalación de dos canales, pero era difícil ver ambas películas al mismo tiempo. Así que conversamos por segunda vez con el editor, Augie Robles, para crear una película experimental de un solo canal. jTodo el proyecto fue experimental!" 4 .

Washoe (septiembre de 1965 - 30 de octubre de 2007) fue un chimpancé común hembra que fue el primer no-humano en aprender a comunicarse usando el Lenguaje de Señas Americano (ASL) como parte de un experimento de investigación sobre la adquisición del lenguaje animal. 2020 . 


\section{CONTEXTO LOCAL Y MARCO LEGISLATIVO}

Considerando las múltiples definiciones y relaciones posibles que establece la investigación artística en la actualidad, junto a todas sus posibilidades expansivas y emancipatorias, ésta aún no tiene un correlato claro en el marco legal que rige actualmente la investigación en Chile. De esta forma, la crisis de la educación formal y el conocimiento disciplinario que constatamos desde las universidades, es también reflejo de un contexto social y cultural que sigue siendo crítico y adverso. Un ejemplo es que el nuevo Ministerio de Ciencia, Tecnología, Conocimiento e Innovación que, pese a que incluye la palabra "conocimiento" en sus declaraciones oficiales, aún considera exclusivamente el sistema científico asociado a su producción. Las lógicas cientificistas imperantes de la carrera académica dificultan situar la carrera artística en un espacio de legitimación académica, que merezca los mismos privilegios de los investigadores de las llamadas "ciencias básicas". Los criterios de excelencia que operan en la investigación son de hecho exportados y aplicados desde estos campos científicos a las artes, humanidades y ciencias sociales, los que por cierto evalúan cantidad de productividad más que procesos (sistema de impacto). Muchas preguntas nos hacemos hoy al respecto: ¿Cómo desde nuestras prácticas podemos ampliar la definición de "conocimiento", permitiendo su entrada al saber artístico en los programas de asignaturas y también en los criterios institucionales de investigación? ¿Cómo invertir la lógica y exportar criterios desde la investigación artística afectando el sistema de investigación nacional? ¿Por qué aún los financiamientos para realizar investigación artística en Chile se organizan en la lógica de la "producción de eventos" y destinados a periodos demasiado acotados de tiempo? Esta última pregunta alude al caso de fondos concursables anuales que dispone el Ministerio de las Culturas, las Artes y el Patrimonio de Chile.

La pintora y académica del Departamento de Artes Visuales de la Universidad Metropolitana de Ciencias de la Educación (UMCE), Macarena Rioseco, ha desarrollado un modelo pictórico bajo el nombre de "minimalismo gestual" a través de ejercicios prácticos fundamentados en las teorías de Deleuze y Guattari (Rioseco 2019). Por su parte, el poeta conceptual y académico del Instituto de Estudios Avanzados, IDEA, de la Universidad de Santiago (USACH), Felipe Cussen, ha señalado que es artificial separar las etapas de desarrollo de un proyecto de investigación y un proyecto de creación (2016). Tanto Rioseco como Cussen reconocen que ambas dimensiones van de la mano y son recíprocas: un proceso artístico surge impulsado por la indagación teórica; y, a su vez, el desarrollo de una reflexión teórica no puede sino alimentarse de dispositivos prácticos. Llevada esta reflexión al marco legal local, nos encontramos con que las bases del concurso al fondo nacional de investigación, Fondecyt, declara expresamente que financia proyectos de "investigación científica y tecnológica”. En Fondecyt no solo no es reconocida la investigación artística sino tampoco todas las prácticas asociadas a la investigación en artes y humanidades (Cussen 2016: 191): exposiciones, curatorías, elaboración de material docente, catálogos de exposiciones, traducciones de textos, entre otros.

A su vez, se puede afirmar que el sistema científico privilegia el individualismo y la competencia, por sobre la investigación asociativa y el establecimiento de redes cola- borativas (Ayala y Gaínza 2020: 9). Bajo el sistema de "proyectos concursables" de la actual ANID (Agencia Nacional de Investigación y Desarrollo), la asociatividad recién puede darse en la etapa de Fondecyt Regular y de manera bastante restringida. En todos estos ámbitos y frente a la concepción de este nuevo ministerio en Chile, la Asociación de Investigadores en Artes y Humanidades (A\&H) ha liderado una serie de iniciativas que pretenden visibilizar las exclusiones aquí comentadas (Ayala y Gaínza 2020). Finalmente dar cuenta que el programa de núcleos milenios de investigación que financia la actual ANID, también se rigen por los criterios científicos-tecnológicos. Cabe mencionar que entre sus proyectos se cuenta solo uno en el ámbito artístico, MAPA, que trabaja la relación entre arte, performatividad y activismo, bajo el principio que el ser/devenir ciudadano consiste en una práctica corporizada que se despliega en el espacio público. El núcleo está dirigido por Milena Grass, de la Escuela de Teatro de la PUC5.
5 Para más información sobre el núcleo milenio MAPA, consultar su sitio web https://www.nmapa.cl 


\section{INSERTO INVESTIGACIÓN ARTÍSTICA \#2}

Serie Fractales (2015-2016)

Macarena Rioseco Castillo 6

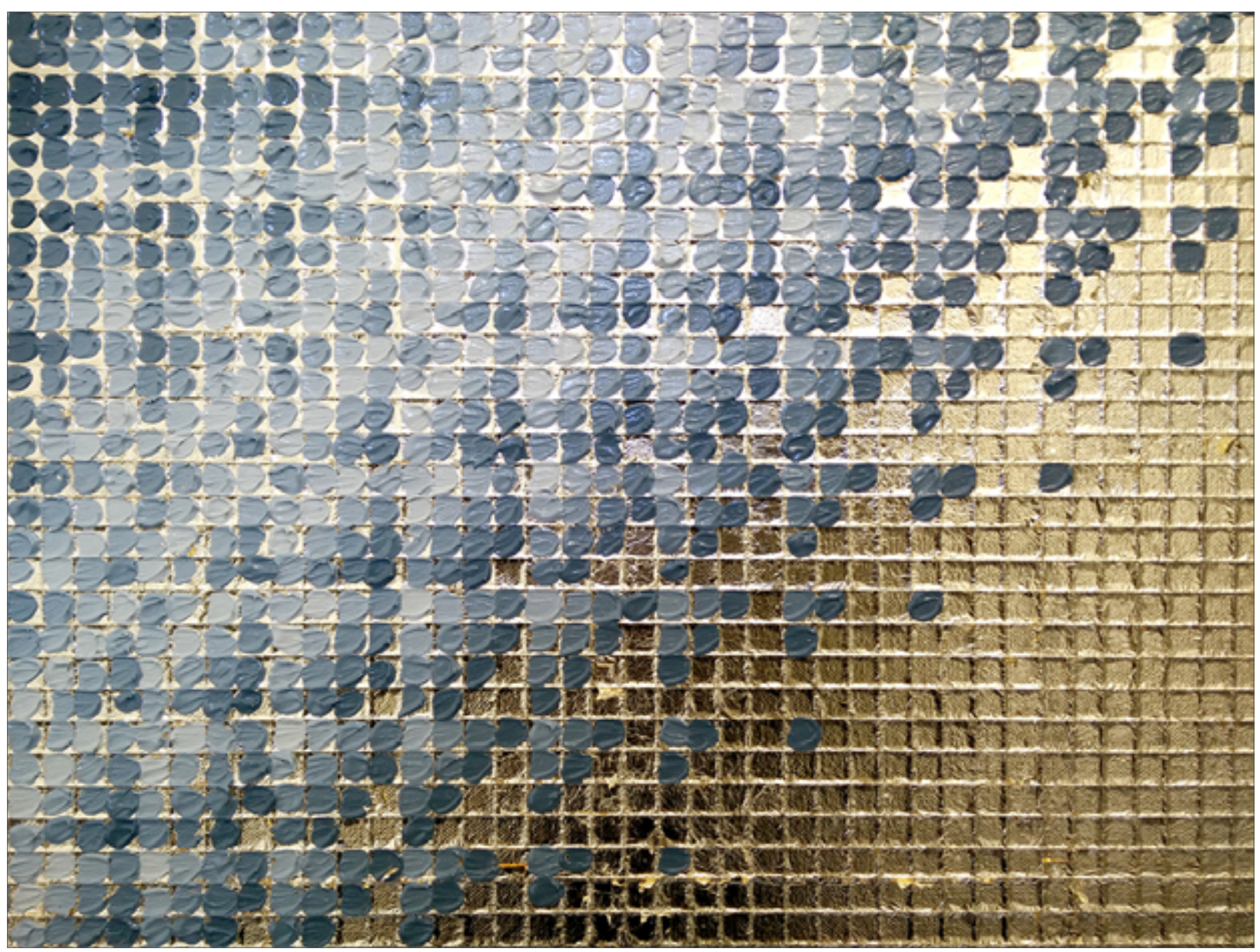

Imagen 2. Cortesía de Macarena Rioseco Castillo

Título: Fractales: Átomos: Ondas (tríptico - detalle en proceso)

Año: 2016

Técnica: Hilos de algodón, dorado a la hoja (aleación de cobre y zinc) y óleo sobre tela.

Dimensiones: Detalle - tríptico: $117 \mathrm{X} 117 \mathrm{~cm}$. (c/u) 


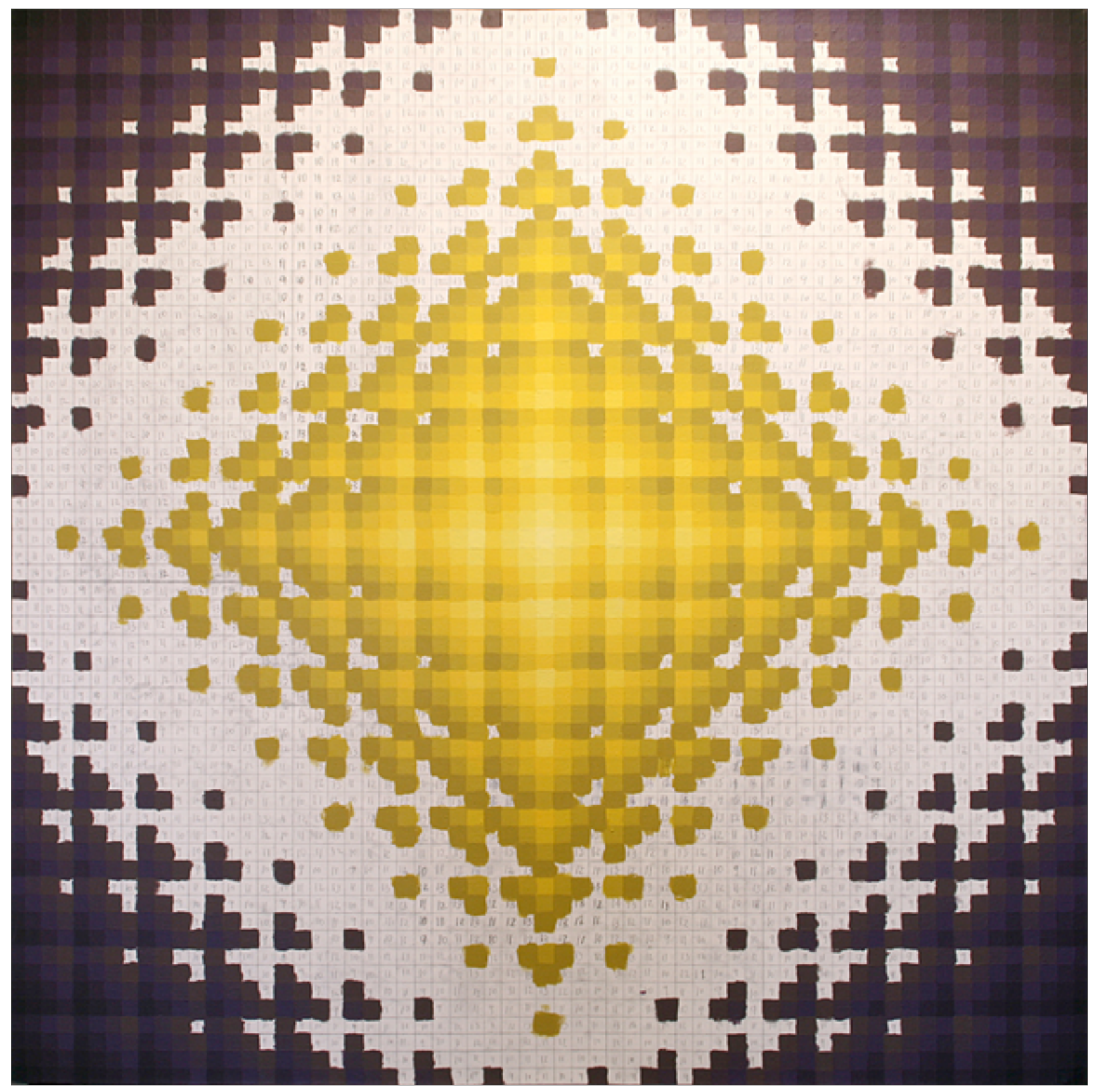

Imagen 3. Cortesía de Macarena Rioseco Castillo

Título: Fractales: Superficies Tejidas Fragmentadas y Lisas (díptico, uno de dos)

Año: 2015

Técnica: Acrílico sobre tela.

Dimensiones: $100 \mathrm{X} 100 \mathrm{~cm}$ 

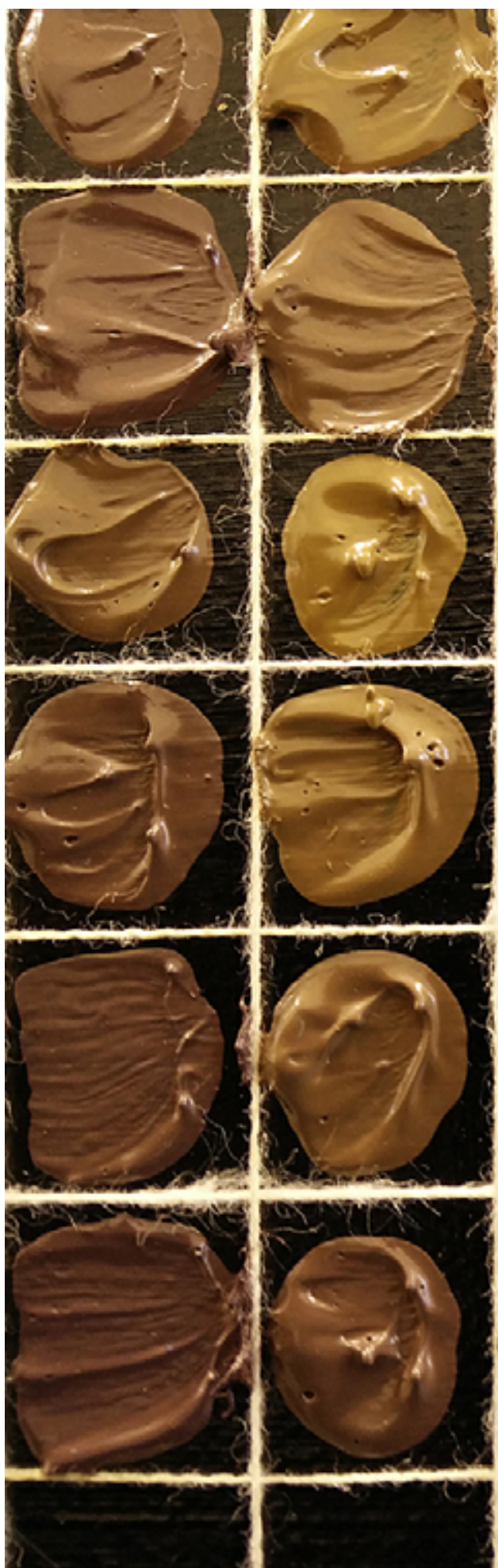

Imagen 4. Cortesía de Macarena Rioseco Castillo

Título: Fractales: Átomos (díptico - detalle en proceso)

Año: 2015

Técnica: Hilos de algodón y óleo sobre tela.

Dimensiones: Detalle - díptico: 96 X $96 \mathrm{~cm}$. (c/u)
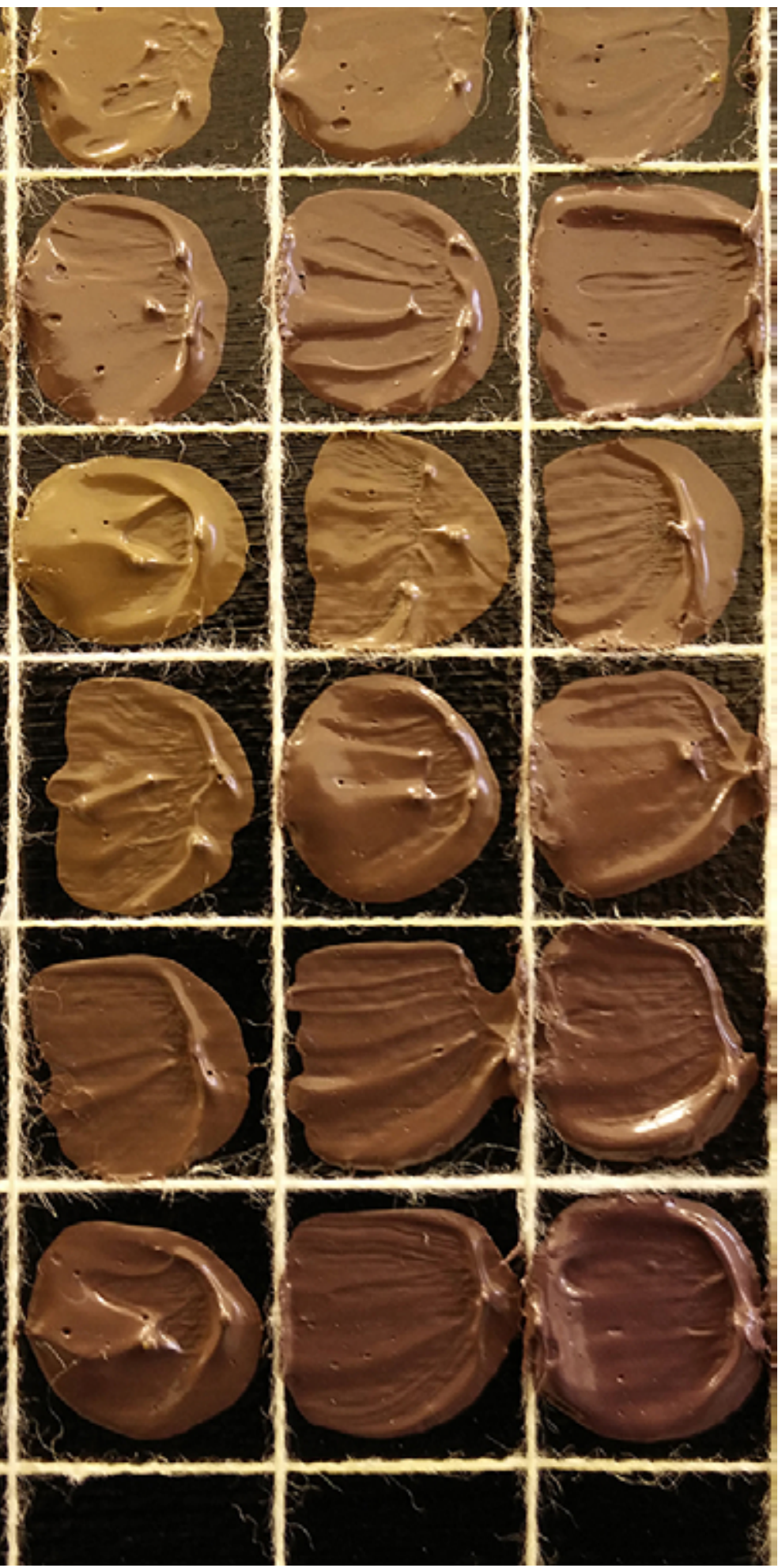
“Mi pintura presenta una reflexión sobre métodos composicionales esencialmente agregados y fragmentados, que se estructuran como acumulaciones múltiples de una unidad monocroma y los cuales se encuentran en piezas tejidas, imágenes de mapa de pixel y estructuras fractales. La transversalidad de este método me incentivó a buscar un territorio en común donde elementos del tejido, píxel y fractales pudieran coexistir mediados por la pintura. La influencia de esas prácticas y medios tradicionales hacen que mis pinturas transmitan una fuerte sensación de proceso y materialidad, mientras que el impacto de la cultura digital se refleja en elementos serializados y repetitivos que le dan a mis trabajos propiedades parecidas a las de objetos hecho por medio de máquinas.

Mi investigación pictórica se estructura en torno a la metodología Investigación basada en la práctica y sigue el propósito de definir un modelo de pintura abstracta basada principalmente en dos marcos teóricos: la filosofía del devenir de Deleuze y Guattari, y el enfoque enactivo de las ciencias cognitivas presentada por Varela, Thompson y Roch. Ambos modelos proponen que la relación entre individuos y el mundo material es dinámica, o sea, presentan una visión donde ninguno precede al otro, sino que más bien forman una relación recíproca donde se co-crean el uno al otro y continuamente cambian y co-evolucionan a través del tiempo. La particular relevancia de las ideas presentadas por estos dos modelos dinámicos, en relación a la forma como yo entiendo y experimento el proceso creativo, me incentivó a integrarlas en mi trabajo para analizar y reflexionar sobre la relación entre individuos y la práctica pictórica.

Un foco principal de mi práctica es el movimiento. Sigue un enfoque post-minimalista centrado principalmente en materiales, el gesto y relaciones de color. La llamo Minimalismo Gestual porque se estructura en torno a repeticiones de un gesto reducido, que es rítmicamente regular y preciso. Este se basa en movimientos repetitivos y mecánicos típicos de técnicas de tejido a mano y en ritmos naturales como son las ondas, vibraciones y la respiración. Experimento la práctica como una meditación, o mantra, que sintoniza con mi cuerpo y, como consecuencia, desencadena afectos calmos.

El fractal además informa la distribución de colores en la retícula de mis pinturas. Esta organización produce patrones cromáticos iterativos y auto-similares entre las imágenes como un total y las partes que las componen, donde las relaciones entre tonos en las pinturas se repiten a diferentes escalas de magnificación. O sea, cada sección cuadrada contiene relaciones de color similares a toda la pintura, así como a secciones más grandes o más pequeñas de ella. Como resultado, ritmos visuales vibrantes parecen surgir de estas composiciones"

Conversación sostenida el 23 septiembre 2020 vía correo electrónico.

\section{APERTURAS DESDE LA UNIVERSIDAD.}

Es preciso destacar que en el aún incipiente campo de la investigación artística en Chile ya comienzan a verse algunos programas de postgrado, centros de investigación y publicaciones periódicas. Han sido tremendamente valiosas las operaciones que estas iniciativas han logrado instalar a propósito de hacer efectiva una investigación que convoca a distintas facultades de las universidades, rompiendo con el criterio disciplinario histórico. Se cree relevante atender a que el desarrollo y validación académica de la investigación artística es un fenómeno global que tiene que ver con los énfasis en investigación, donde su marco referencial es el Plan de Bolonia guiado por el Manual Frascati ${ }^{8}$.

En términos de programas habría que mencionar el Magíster y Doctorado en Artes de la Pontificia Universidad Católica de Chile (PUC), enfocado en la investigación basada en la práctica, aunque dichas prácticas deben tener una matriz centrada en alguna disciplina de la Escuela donde se enseña (Artes Visuales, Artes Escénicas y Música). Por su parte, el Doctorado en Estudios Interdisciplinarios de la Universidad de Valparaíso, reconoce la investigación artística como eje productivo de saber entre sus diferentes líneas de investigación. Este Doctorado, según se declara en su documentación, "posee una perspectiva pedagógica novedosa que considera la actividad de investigar como un oficio y que apunta a la producción de conocimiento de carácter situado, basado en una actividad colectiva y colaborativa" . De esta manera, el programa incorporaría las metodologías emergentes del practice-based research, que trascienden el llamado campo artístico, incorporando el activismo, la antropología, la conversación, los rituales, la oralidad, entre muchas otras prácticas. Es al alero de este programa académico que ha nacido en 2015 la presente revista Panambí.

Cabe mencionar también el Magíster en Arte, Pensamiento y Cultura Latinoamericanos del Instituto de Estudios Avanzados (IDEA) de la Universidad de Santiago de Chile que, pese a la tradición científica del instituto, hoy admite la posibilidad de producir tesis "mixta" o basadas en prácticas. En este contexto, entrega una mirada integral e interdisciplinaria, abordando las distintas áreas de las humanidades y considera el arte como una expresión cultural inherente a las sociedades. Si bien el programa está dirigido a personas formadas en las distintas disciplinas de las humanidades, artes y ciencias sociales, es posible acoger a un postulante de otra área como la ingeniería. "No es un programa cerrado, sino que al promover y favorecer la interdisciplinariedad, también estaría abierto a personas que provienen de otras áreas del saber", explica la Dra. Carolina Pizarro ${ }^{10}$.

El Manual de Frascati es un documento que establece la metodología para la recopilación de estadísticas sobre investigación y desarrollo. El Manual fue preparado y publicado por la Organización para la Cooperación y el Desarrollo Económico (OCDE). El manual da definiciones para: investigación básica, re-investigación aplicada búsqueda, investigación y desarrollo, etc. También organiza el campo de la ciencia en categorías principales y subcategorías.

Ver la presentación del programa en: https://postgrados.uv.cl/ doctorado/doctorado-en-estudios-interdisciplinarios-sobre-pensamientocultura-y-sociedad. Recuperada 20 de septiembre 2020.

10 Ver entrevista en: http://www.usach.cl/news/magister-artepensamiento-y-cultura-latinoamericanos-inicia-su-ano-academico. 
En esta línea también se inscribe el programa de Magíster en Prácticas Artísticas Contemporáneas (MG PAC) de la Universidad Finis Terrae, que desde el año 2018 ha venido construyendo una plataforma de investigación colectiva que reúne a docentes de la universidad que ejercen en distintas Escuelas (Arte, Teatro, Arquitectura, Diseño, Publicidad y Literatura). En este programa académico se instala una línea de taller transversal que no se divide en especialidades ni menciones, bajo la premisa de que es necesario el diálogo y retroalimentación entre los estudiantes para el desarrollo de sus proyectos. En sus dos versiones, el programa ha operado como plataforma para el desarrollo de investigaciones prácticas asociadas a la educación artística, la memoria colectiva, la cultura visual, el feminismo, la salud, entre otros. Los estudiantes son acompañados en este proceso por los profesores de las diferentes asignaturas en la línea de taller, instrumental y teórica.

Finalmente, cabe mencionar en esta revisión al Magíster en Investigación y Creación Artística de la Universidad Mayor, liderado en su momento por la académica y bailarina Jennifer McColl, que aunque hoy descontinuado, se destacó porque permitió a sus estudiantes desarrollar investigaciones en intensivos talleres prácticos de carácter situado con artistas invitados, tales como Ingrid Wildi y Juan Castillo, dimensión que lo distancia de programas de postgrado de corte teórico en artes, como por ejemplo, el de la Universidad de Chile.

\section{INSERTO INVESTIGACIÓN ARTÍSTICA \#3.}

novela / VIA (2018), Fernando Pérez Villalón ${ }^{11}$.

Link video: https://www.youtube.com/watch?v=F-BplBMlj8E\&feature =youtu.be\&fbclid $=$ IwAR35V_hz5E5Elo6 AxOdF_ ZXD3-FR18lifXqUBOEvqvSc4sCA9Xy1Swf9EJQ

"Hace ya tiempo que trabajo con lecturas colectivas y simultáneas de texto. Me interesa mucho la tensión entre el texto como un objeto comprensible y el galimatías de voces como superficie sonora desprovista de contenido semántico inteligible, así como la desaparición de la voz del autor como única voz asociada al poema y su reemplazo por un conjunto de voces diversas. Entonces en el caso de novela / VIA, que es un libro-objeto publicado en 2018 por la editorial Libros del Pez Espiral, el texto existe en formato libro-objeto y en formato audiovisual. En uno puede leerse de manera silenciosa y lineal, en otro, experimentarse como un conjunto sonoro de fragmentos sueltos, dispersos en varias voces $y$ acompañados por imágenes asociadas a los múltiples relatos con los que el texto dialoga.

El libro-objeto novela / VIA consiste en un sobre plegado (11,5 X 15,5), sellado con lacre, que contiene dos folletos: al abrir el sobre, a la izquierda está VIA y a la derecha novela, un conjunto de poemas en verso libre que imitan el estilo de los libros de aventura de autores como Karl May, Emilio Salgari o Julio Verne que leía en mi infancia y juventud. novela es una hoja plegada en 8 , con un corte al centro que permite formar páginas (en el típico plegado del fanzine en hoja de carta). Por un lado están el título, los poemas y algunas ilustraciones, por otro lado hay una obra visual, un dibujo de Marcos Sánchez que recrea la operación de los textos, una inmersión en el universo de las novelas de aventuras plagadas de peligros al acecho, exploradores, corsarios, decorados exóticos, mujeres misteriosas y suspenso narrativo. Me interesaba no tanto burlarme de lo envarado y pomposo de los textos, sino recuperar su atmósfera enrarecida en la que me formé como lector, al mismo tiempo que intentar producir en un poema una sensación narrativa, en textos que parecen sacados de un conjunto más extenso pero quedan inconclusos. Para el lanzamiento, en la Librería del GAM, se hizo una lectura susurrada al oído de los asistentes de los poemas de novela, pidiéndoles que los repitieran como en un juego del teléfono y acompañando la lectura oral con un video realizado por Pablo Fante a partir de imágenes fijas (seleccionadas y fotografiadas por mí a partir de las adaptaciones al formato de cómic de novelas de los autores parodiados) y una pista sonora consistente en un collage de músicas de película encontradas en youtube. Para el video, le pedí grabar cada uno de los siete poemas a José Burdiles, Felipe Cussen, Federico Eisner, Pablo Fante, Francisca García, Jessica Pujol y Macarena Urzúa”. ${ }^{12}$ 


\section{Conclusiones.}

El texto abre la discusión sobre investigación artística, que más que una "estrategia para operar en la academia" (que ha sido una crítica más o menos común en el medio artístico), lo hemos planteado al revés, como una estrategia para ampliar la academia y permitir la convivencia de discursos en torno a la producción de conocimiento y como este pudiese rendir a la producción de sentido. Generalmente hay artistas que desconfían de la noción de "metodología", no están de acuerdo con ciertas ideas procidementales que son formalizadas como tal. La figura del artista es escéptica, aquella condición se da dentro y fuera de la academia. Desde ese lugar uno podría hacer el ejercicio de invertir la pregunta. Según un artículo publicado en mayo del 2016 en la revista científica Nature, más de un $70 \%$ de los experimentos son irrepetibles (Baker). ¿Son los científicos artistas también? Los artistas poseen una visión refractaria y es contradictorio decir que no utilizan un método de investigación, porque en el fondo sí lo hacen, sobre todo en el ámbito comercial, cuando sistematizan ciertas técnicas de forma repetitiva, dado a ciertas tendencias de mercado.

Este texto pretende no solo funcionar como una revisión y argumentar que el arte produce conocimiento al interior de la academia, sino que también desplegar la práctica artística como investigación hacia otros espacios como los centros de residencia o de investigación, las plataformas, las redes y/o a las publicaciones. En un sentido económico se abre una vieja discusión: ¿de qué vive el artista? o ¿cómo se deberían financiar estas prácticas artísticas basadas en la investigación?, ¿cómo se argumentaría y qué tipo de financiamiento se daría? ¿quién lo asignaría? ¿el Estado? ¿un mecenas? ¿los coleccionistas?, ¿la academia?. Y a continuación, ¿qué pasaría con la propuesta desarrollada una vez concretada la investigación? ¿quedaría en manos de colecciones privadas? ¿integraría centros de investigación para un proyecto de más largo aliento? ¿quedarían los resultados de investigaciones en las colecciones de museos, bibliotecas?

En torno a la identidad del artista y su profesionalización, mantenemos como autores una evaluación mixta en donde reconocemos algunos pro y contra. En el primer caso, la investigación artística operaría como un posicionamiento profesional de los artistas en general, que permite equipararlos a cualquier otro investigador profesional de los distintos campos disciplinarios y científicos. Ello permitiría desentenderse de la apreciación generalizada de que los y las artistas "somos raros" o "distintos". Por otra parte, con esta condición profesionalizante se tendería a una cierta integración en el campo de las artes, en la medida que se podría debilitar esa división de clase que trama el mercado y la venta de objetos artísticos: el artista que produce artesanías y las comercializa de mano directa, versus, el artista que participa de las redes de coleccionistas o vende a través de galerías. En cuanto a los riesgos de la condición profesionalizante ha sido que el artista contemporáneo se centra en una hiper profesionalización de su trabajo, desarrolla una mirada fordista de la producción que es facilitada por una red la circulación centrada en el poder de poseer. El dilema de ser artista y ser "profesional" se encarna en una lógica productiva del capitalismo y de la cultura neoliberal que impulsa la competencia descarnada entre pares y al camino en solitario que considera una alta competitividad.

La práctica artística va más allá de las teorías que afectan la práctica o qué conceptos teóricos se desarrollan desde la práctica particular. Esta consideración ha sido siempre impuesta desde la academia. Una dificultad común que venimos constatando en el posgrado es que los artistas no tienen formación en escritura ni en lectura crítica. Principalmente podemos constatar que en las escuelas de artes visuales, la práctica de escritura siempre ha sido subordinada al "hacer" del taller, y tal como hemos comentado en páginas anteriores, relegada al último semestre de licenciatura en donde los estudiantes de arte deben desarrollar sus memorias sin ninguna preparación previa. Escribir implica reflexión teórica y esa reflexión impacta los procesos artísticos, tal como ya comentamos, en una relación de ida y vuelta. La escritura y la lectura son prácticas que desarrollan la auto-conciencia de los procesos artísticos. Estas también se aprenden -como cualquier otra práctica expresiva-y deben formar parte del trayecto formativo de cualquier artista para motivar la reflexión y para permitir su propia inscripción en un campo cultural y artístico. Asimismo se hace fundamental el desarrollo de la práctica de la lectura en torno a textos teóricos, críticos o filosóficos que expanden el horizonte de referencia respecto de las prácticas y sentires de la experiencia artística. El manejo de herramientas intelectuales ya a nivel de pregrado, facilitaría sin duda el desarrollo de proyectos de investigación y sobre todo la capacidad de formulación de preguntas y conexiones. Es decir, se trata sin duda de una transformación profunda y de largo aliento.

Existe además un vínculo entre creación artística y actividad pedagógica, todos los artistas en las universidades son también docentes (co-construcción con sus propios alumnos), por lo que investigadores que ingresen a esta línea podrían preguntarse ¿cómo evalúan ellos a sus estudiantes? ¿cómo evalúan ellos su práctica? ¿por qué lo hacen? y darse el tiempo de reflexionar ¿quiero imponer una geneología de lo que conozco? ¿cómo valoro el trabajo del otro?

Del mismo modo que en un programa de postgrado científico tradicional, la admisión a un programa de postgrado de investigación artística basado en práctica requiere de la presentación por parte de los postulantes interesados de un proyecto claro en base a un formato. El breve periodo de duración de un programa magister - dos años es la convención- se traduce en un plan de estudios en los que el artista debería no solo desarrollar su propio proyecto, sino dejarse nutrir por talleres que aportan herramientas metodológicas tanto en la dinámica grupal como desde docentes que proponen diversos laboratorios de investigación artística. Se entiende que estos talleres son siempre de tipo experimental con bases metodológicas propuestas por los docentes y utilizando diversas herramientas. En el curso del primer año, lo usual sería que las investigaciones den pie a transformaciones muchas veces sustanciales en cuanto al foco de interés y las metodologías utilizadas, transformaciones que se darían en base a una integración de nuevos elementos y adaptación de otros.

Las fórmulas académicas de programas de postgrado tradicionales (claustro académico, plan de estudio con 
asignaturas fijas, escritura de tesis, comisión de evaluación de tesis, comisión de examen final) puede que no sean del todo productivas en un programa de investigación artística. En estos casos es fundamental la presencia de grupos colegiados de profesores y profesoras que desarrollen una relación apegada e íntima en torno a los procesos artísticos, no solo la supervisión técnica. Por tratarse justamente de metodologías no tradicionales de investigación, será necesario que el trabajo docente se comprenda como un acompañamiento durante el trayecto, puesto que evaluaciones o comisiones finales "en frío" no permiten la afectación de que suponen todos los procesos y por ende, inhabilitan la propuesta misma del programa. Por lo tanto nos parece fundamental que exista un seguimiento cercano donde tanto estudiantes como académicos invitados, desarrollen una co-construcción de manera abierta donde exista una experimentación de carácter epistemológico, diferenciándose así de los programas tradicionales de postgrado que generalmente no validan dicha instancia. 
ADJEI, S. K. (2020). The philosophy of art in Ewe Vudu Religion. En Doherty, C. (ed) How does artistic research decolonize knowledge and practice in Africa. Johanesburg: Wits University.

Ayala, M.; Gaínza, C. (EDS.) (2020). La batalla de las artes y humanidades. Archivo 2016-2019. Santiago: Asociación de Investigadores en Artes y Humanidades.

BAKER, MONYA (25 MAYO 2016). 1500 Scientist Lift the Lid on Reproductibility. Nature n- 533, Londres, 452-454. DOI:10.1038/533452a

Barone, T.; Elliot, E. (2012). Art Based Research. Londres: Sage Publications.

Borgdorff, H. (2011). The Production of Knowledge in Artistic Research. En: Biggs, M.; Karlsson, H. (eds.). The Routledge Companion to Research in the Arts. Londres: Routledge, 44-66.

CARTER P. (2004). Material Thinking: The Theory and Practice of Creative Research. Melbourne: Melbourne University Publishing.

(2011). Interest: the Ethics of Invention. En: Barrett, E.; Bolt, B. (eds.). Practice as Research: Approaches to Creative Arts Enquiry. Londres: IB Tauris, 23-34.

Cazeaux, C. (2018). Art Research and Philosophy. Londres: Routledge.

Celedón Bórquez, G. (2020). ¿Qué significa conocimiento en la investigación artística?. En: Benavente, C. (ed.). Coordenadas de la investigación artística: sistema, institución, laboratorio, territorio. Viña del Mar: Universidad de Valparaíso / CENALTES ediciones, 29-53.

Contreras, M. J. (2013). La práctica como investigación: nuevas metodologías para la academia latinoamericana. Poiésis 21-22. Río de Janeiro: Revista do Programa de Pós-Graduação em Estudos Contemporâneos das Artes Universidade Federal Fluminense, 71-86.

(2018). "Santiago, 10 th January 2018. Dear members of the Society for Artistic Research(...)”. Society for Artistic Research (Correspondents). Recuperado de https://societyforartisticresearch.org/correspondents/maria-josecontreras-santiago/

CUSSEN, F. (2016). "Correcciones: práctica artística como investigación como quien no quiere la cosa". Panambí. Revista de Investigaciones Artísticas 3. Valparaíso: Universidad de Valparaíso, 189-206.

DoherTY, C. (ED) (2020). How does artistic research decolonize knowledge and practice in Africa. Johanesburg: Wits University.

EISNER, E. (2006). Does Arts-Based Research have a Future?. Inaugural Lecture for the First European. Studies in Art Education 43 (1). Alexandria, VA: National Art Education Association, 9-18.

Emerling, J. (2019). These on the Concept of Research. En: de Assis, P.; D' Errico L. (eds.). Artistic Research: Charting a Field in Expansion. Lanham, MD: Rowman \& Littlefield Publishers, 12 - 26

Hannula, M., Suoranta, J., Vadén, T. (2005). Artistic Research: Theories, Methods and Practices. Gothenburg: University of Gothenburg.

Kellert, S. H.; Longino, H.; Waters, K. C. (2006). Scientific Pluralism. Studies in Philosophy of Science Vol XIX. Scientific Pluralism. Minneapolis, MN: Minnesota University. 
Knowels, G. J.; Cole, A. (2007). Handbook of the Arts in Qualitative Research. Perspectives, Methodologies, Examples, and Issues. Londres: Sage Publications.

Leddy, T. (Verano 2020). Dewey's Aesthetics. En: Zalta, Edward N. (ed.). The Stanford Encyclopedia of Philosophy. Stanford, CA: Stanford University.

Lincoln, Y. S.; Denzin N. K. (2003). The Revolution in Presentation. En: Lincoln, Y. S.; Denzin, N. K. (eds.). Turning Points in the Qualitative Research. Tying Knots in a Handkerchief. Walnut Creek, CA: AltaMira Press, 375 - 378

Nieto, I.; Velasco, M. (2016). Ciencia abierta. Singularidad e irrupción en las fronteras de la práctica artística. Santiago: Adrede Editores.

Rheinberger, H. J. (1998). Experimental Systems, Graphematic Spaces. En: Lenoir, T. (ed.). Inscribing Science: Scientific Texts and the Materiality of Communication. Stanford, CA: Stanford University Press, 285-303.

Rıoseco, M. (2019). Minimalismo gestual: desarrollando un modelo pictórico a la luz de teorías de Deleuze-Guattari y enactivas. En: Villegas, I.; Espinoza, D. (eds.). La creación artística en artes visuales en Chile y sus formas de investigación: el caso del sistema universitario. Santiago: LOM, 123-147.

Savin-Baden, M.; Wimpenny, K. (2014). A Practical Guide to Arts-related Research. Rotterdam/Boston/Taipei: Sense Publishers.

Schwab, M. (ED.) (2013). Experimental Systems Future Knowledge in Artistic Research. Lovaina: Universitaire Pers Leuven.

Sormani, P.; Garbone, G.; Proska, G. (2019). Practicing Art Science. Londres: Routledge.

Sullivan G. (2005). ArtPractice as Research. Inquiryin Visual Arts. Sage Publications. (2010). Art Practice as Research. Inquiry in Visual Arts. Londres: Sage Publications.

Wilson M.; VAn RuIten, S. (eds.) (2013). SHARE HandBook for Artistic Research. Amsterdam: ELIA. 